\title{
Flow-Induced Surface Charge Heterogeneity in Electrokinetics due to Stern-Layer Conductance Coupled to Reaction Kinetics
}

\author{
B. L. Werkhoven, ${ }^{1}$ J. C. Everts, ${ }^{2}$ S. Samin, ${ }^{1}$ and R. van Roij ${ }^{1}$ \\ ${ }^{1}$ Institute for Theoretical Physics, Center for Extreme Matter and Emergent Phenomena, Utrecht University, \\ Princetonplein 5, 3584 CC, Utrecht, The Netherlands \\ ${ }^{2}$ Department of Physics, Faculty of Mathematics and Physics, University of Ljubljana, Jadranska 19, 1000 Ljubljana, Slovenia
}

(Received 29 March 2018; revised manuscript received 18 May 2018; published 29 June 2018)

We theoretically study the electrokinetic problem of a pressure-induced liquid flow through a narrow long channel with charged walls, going beyond the classical Helmholtz-Schmolukowski picture by considering the surprisingly strong combined effect of (i) Stern-layer conductance and (ii) dynamic chargeregulating rather than fixed surface charges. We find that the water flow induces, apart from the well-known streaming potential, also a strongly heterogeneous surface charge and zeta potential on chemically homogeneous channel walls. Moreover, we identify a novel steady state with a nontrivial 3D electric flux with 2D surface charges acting as sources and sinks. For a pulsed pressure drop our findings also provide a first-principles explanation for ill-understood experiments on the effect of flow on interfacial chemistry [D. Lis et al., Science 344, 1138 (2014)].

DOI: 10.1103/PhysRevLett.120.264502

The flow of water along a solid surface such as glass, rock, or an electrode is of profound interest in fields as diverse a geosciences (rivers, erosion) [1], oil-field engineering (enhanced oil recovery) [2], and micro- and nanofluidics. The Poiseuille flow through a long channel due to a pressure drop between inlet and outlet is a textbook example, in which the stationary Navier-Stokes equation with no-slip boundary conditions on the channel surface gives rise to a parabolic flow profile (represented in Fig. 1) that is proportional to the pressure drop. In many cases relevant for, e.g., microfluidics and blue-energy harvesting $[3,4]$, however, a liquid flow induces a much richer phenomenology, often due to surface charges on the channel walls that interact with the ionic species in the liquid. In such a channel an applied pressure drop does not only induce a fluid flow but also a net electric current due to advection of the so-called electric double layer (EDL), which is the diffuse layer of mobile ions that screen the electrode in the nanometer vicinity of the charged surface. In closed-circuit conditions this so-called "streaming current" can persist in a stationary state, but in open-circuit conditions it leads to the buildup of net charge and hence a potential difference between the outlet and the inlet of the channel, the so-called "streaming potential" $\Delta \Phi_{S}$ derived long ago by Helmholtz [5] and Smoluchowski [6], as

$$
\Delta \Phi_{S}=\frac{-\zeta \epsilon}{\eta G} \Delta p
$$

Here $\zeta$ is the (zeta) potential at the slipping planes, $\epsilon$ and $\eta$ the dielectric permittivity and the shear viscosity of the liquid, respectively, and $\Delta p$ the pressure drop that drives the Poiseuille flow. The total channel conductivity $G=$ $G_{b}+2 G_{s} / H$ of a channel of height $H$ is well known to consist not only of a bulk contribution $G_{b}$ but also of two surface contributions $G_{s} / H$ to account for conduction processes close to the channel surfaces [7].

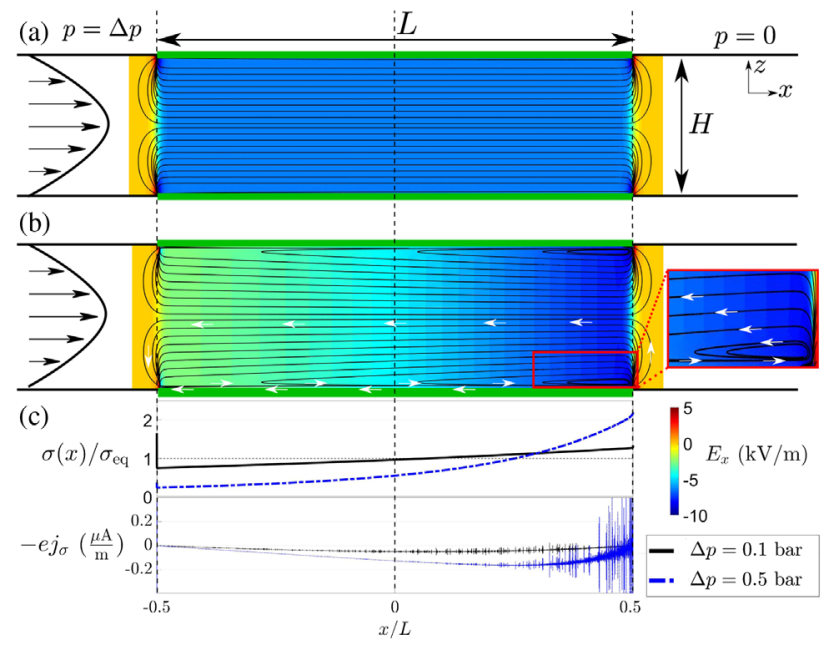

FIG. 1. Streamlines of the net charge flux and color map of the tangential electric field $E_{x}$ near the charged surfaces (green stripes) of a rectangular channel with a pressure drop $\Delta p=0.5$ bar between inlet and outlet at $x= \pm L$, (a) with vanishing Sternlayer conduction $\left(D_{s}=0\right)$ resulting in a fixed surface charge of $-e \sigma_{\mathrm{eq}}=-0.069 e / \mathrm{nm}^{2}$ that mimics silica at $p \mathrm{H}=6.5$, and in (b) with nonzero Stern-layer conductance and our dynamic charge regulation model. (c) Flow-induced heterogeneous surface charge density $\sigma(x)$ and surface charge flux $-e j_{\sigma}$ for $\Delta p=0.1$, 0.5 bar for the parameters of the case of (b). 
The dimensionless Duhkin number $\mathrm{Du}=G_{s} / G_{b} H$ characterizes the relative importance of the surface-to-bulk conduction [7]. It is important to realize that $G_{s}=G_{s}^{d}+G_{s}^{S}$ not only contains a contribution $G_{s}^{d}$ from the relatively high density of charge carriers in the diffuse part of the EDL, as first recognized by Bikerman in 1933 [8,9], but also a contribution $G_{s}^{S}$ from the quasi-2D Stern layer where the surface charges reside [10]. In fact, a substantial body of literature exists that not only confirms the finite charge mobility in the Stern layer for different types of (insulating) materials such as PMMA, silica or clay [11-16], but even that the in-plane charge mobility is comparable to the mobility of simple ions in bulk electrolytes $[11,17,18]$. The lateral conductance in the Stern layer is the first key ingredient of this Letter.

Equation (1) stems from a linear-response analysis, in which the prefactor $-\zeta \epsilon / \eta G$ is assumed to be a constant for a given channel and transported fluid. Motivated by inherently heterogeneous biological surfaces and by microfluidic applications with patterned electrodes, extensions towards periodic [19] and steplike [20] variations of $\zeta$ were considered. Heterogeneity of $\zeta$ not only leads to normal components of the ionic fluxes $[19,20]$, but also to the notion of the so-called healing length $\ell=G_{s} / G_{b}=H \mathrm{Du}$ as the governing lateral length scale [20]. However, in this Letter we will for the first time show that defect-free and unpatterned surfaces, charged over a finite length, can exhibit flow-induced heterogeneities with the surface charge density and the zeta potential varying over the full length of the charged surface, even if Du $\ll 1$. Here one should realize that most surfaces in contact with water do not have a fixed charge but obtain their net charge by regulation processes, in which, for instance, a fraction $f$ of the neutral surface groups SC dissociates into a covalently bound negatively charged surface group $\mathrm{S}^{-}$and a released cation $\mathrm{C}^{+}$. The reaction $\mathrm{SC} \rightleftharpoons \mathrm{S}^{-}+\mathrm{C}^{+}$is characterized by an equilibrium constant $K$, which together with the $\mathrm{C}^{+}$ concentration at the surface $\rho_{C, s}$ determines the equilibrium Langmuir desorption isotherm $f=\left(1+\rho_{C, s} / K\right)^{-1}$ [21-23]. Although the importance of charge regulation was indeed recognized in earlier works on the electrophoresis of colloidal particles, the underlying equilibrium Langmuir desorption isotherm has so far always been assumed $[24,25]$. In this Letter we will introduce out-of-equilibrium charge regulation as a second key ingredient, in which the rates of adsorption $\left(k^{\text {ads }}\right)$ and desorption $\left(k^{\text {des }}\right)$ play a key role individually rather than only their ratio $K=k^{\text {des }} / k^{\text {ads }}$. In fact, by tuning the chemical rates to the reaction-limited regime, we will see that our theory provides a natural first-principles explanation for puzzling recent experiments that show a profound influence of a fluid flow on the interfacial chemistry [26], provided Stern-layer conduction and out-of-equilibrium charge regulation are taken into account simultaneously. We expect that this intricate interplay between dynamic charge regulation and Stern-layer conduction will play an equally important role in many nanoflow problems of recent interest [4,27-29].

The system we consider in this Letter, sketched in Fig. 1, consists of two bulk aqueous reservoirs connected by a wide rectangular channel of length $2 L$ and height $H$, with lateral and normal Cartesian coordinates $x \in[-L, L]$, $z \in[0, H]$, and with translational invariance in the lateral $y$ direction. The reservoirs contain three monovalent ionic species labeled by $i=+,-, C$, with valency $z_{+}=z_{C}=$ $-z_{-}=1$ and with bulk concentrations $\rho_{i, b}$, satisfying neutrality $\sum_{i} z_{i} \rho_{i, b}=0$. The Debye screening length is given by $\lambda_{D}=\sqrt{\epsilon k_{B} T / e^{2} \sum_{i} z_{i}^{2} \rho_{i, b}}$, with $k_{B}$ the Boltzmann constant, $T$ the temperature, and $e$ the proton charge.

We denote the time- and position-dependent ionic density profiles and fluxes (actually flux densities) by $\rho_{i}(\mathbf{r}, t)$ and $\mathbf{J}_{i}(\mathbf{r}, t)$, respectively, the electric potential by $\psi(\mathbf{r}, t)$, the (identical) surface charge density in the planes $z=0$ and $z=H$ by $-e \sigma(x, t)$, and the fluid velocity profile by $\mathbf{u}(\mathbf{r}, t)$. The ion flux $\mathbf{J}_{i}$ is composed of diffusive, conductive, and advective contributions, and is related to $\partial_{t} \rho_{i}$ by the continuity equation. The Poisson equation accounts for Coulomb interactions, and the incompressible Navier-Stokes equation, including an electric body force and a pressure gradient $\nabla p$, describes the fluid flow. Collecting all this we obtain the well-known PoissonNernst-Planck-Navier-Stokes (PNPNS) equations [30] with Gauss's law and no-slip boundary conditions:

$$
\begin{aligned}
\frac{\partial \rho_{i}}{\partial t} & =-\nabla \cdot \mathbf{J}_{i} ; \quad \mathbf{J}_{i}=-D\left(\nabla \rho_{i}+\frac{e z_{i} \rho_{i}}{k_{B} T} \nabla \psi\right)+\rho_{i} \mathbf{u} ; \\
m \frac{\partial \mathbf{u}}{\partial t} & =-m(\mathbf{u} \cdot \nabla) \mathbf{u}-\nabla p+\eta \nabla^{2} \mathbf{u}-\sum_{i} z_{i} e \rho_{i} \nabla \psi \\
\nabla \cdot \mathbf{u} & =0 ; \quad \nabla^{2} \psi=-\frac{e}{\epsilon} \sum_{i} z_{i} \rho_{i} ; \\
\mathbf{u}_{s} & =0 ; \quad \mathbf{n}_{s} \cdot \nabla \psi_{s}=\frac{e \sigma}{\epsilon} .
\end{aligned}
$$

Here $D$ is the diffusion constant, assumed to be equal for all ion species, and $m$ is the mass density of water. Throughout this Letter the subscript $s$ denotes a surface quantity; e.g., $\mathbf{u}_{s}$ is the fluid velocity at the surface and $\mathbf{n}_{s}$ is the unit surface normal pointing into the water.

The standard PNPNS equations [Eq. (2)] are to be contrasted with the novel boundary conditions that we impose in this Letter, where we describe regulation of the surface charge $\sigma$ combined with Stern-layer conductance [31]. The bottom and the top surfaces each contain an identical, chemically homogeneous patch for $x \in$ $[-L / 2, L / 2]$ that can become negatively charged due to the desorption reaction $\mathrm{SC} \rightleftharpoons \mathrm{S}^{-}+\mathrm{C}^{+}$. Therefore, a nonvanishing cationic counterion flux $-\mathbf{n}_{s} \cdot \mathbf{J}_{C, s}(x)$ entering the surface is possible, whereas the other two ionic species \pm are chemically inert and satisfy the no-flux boundary 
conditions $\mathbf{n}_{s} \cdot \mathbf{J}_{ \pm, s}=0$. Within the Stern layer we introduce the lateral (surface) current $-e j_{\sigma}(x)$, satisfying the continuity equation for the surface charge density as

$$
\frac{\partial \sigma}{\partial t}=-\frac{\partial j_{\sigma}}{\partial x}+\mathbf{n}_{s} \cdot \mathbf{J}_{C, s},
$$

which explicitly couples the 3D flux of cations as a source term for the 2D surface density. We describe the net flux of $\mathrm{C}^{+}$towards the surface in terms of simple reaction kinetics with an adsorption flux $k^{\text {ads }} \sigma \rho_{C, s}$ and a desorption flux $k^{\operatorname{des}}(\Gamma-\sigma)$, with $\Gamma$ the total number of chargeable sites per unit area. Additionally, we assume a Nernst-Planck-like equation for $j_{\sigma}$, with a diffusive and a conduction contribution, where the former is modified to account for forbidden multiple adsorption and desorption on a single site [35]:

$$
\begin{gathered}
j_{\sigma}(x)=-D_{s}\left(\frac{1}{1-\sigma / \Gamma} \frac{\partial \sigma}{\partial x}-\frac{e \sigma}{k_{B} T} \frac{\partial \psi_{s}}{\partial x}\right), \\
-\mathbf{n}_{s} \cdot \mathbf{J}_{C, s}=-k^{\operatorname{des}}(\Gamma-\sigma)+k^{\mathrm{ads}} \sigma \rho_{C, s},
\end{gathered}
$$

where $D_{s}$ is the surface diffusion constant, which we have seen to be comparable to the bulk diffusion coefficient $D$. If we impose static equilibrium conditions, in particular $\mathbf{J}_{C}=0$, Eqs. (3)-(5) reduce to the standard Langmuir desorption isotherm where $\sigma / \Gamma$ equals the fraction $f$ of charged sites introduced above [36]. In the case of a pressure-induced flow, however, the streaming potential generates an in-plane electric field component $\partial_{x} \psi_{s}$, which according to Eq. (4) not only drives a finite $j_{\sigma}$ if $D_{s} \neq 0$, but for a charge-regulating surface also a finite $\mathbf{n}_{s} \cdot \mathbf{J}_{C, s}$ and a surface heterogeneity $\partial_{x} \sigma$ according to Eqs. (3) and (5). As a consequence the zeta potential $\zeta(x)=\psi(x, 0)-$ $\psi(x, H / 2)$ becomes heterogeneous too, and hence a nontrivial self-consistency problem emerges in which the streaming potential not only determines $\zeta(x)$ but also depends on it [see, e.g., Eq. (1)]. Interestingly, this flowinduced surface heterogeneity does not require relatively narrow channels or high Du.

We solve the set of nonlinear equations (2)-(5) numerically using the Finite-Elements software COMSOL MULTIPHYSICS. For computational reasons we take at each side of the chargeable surface an uncharged patch of length $L / 2$ to allow entrance and exit effects on the fluid flow driven by a pressure drop $\Delta p$ to essentially die out [37]. Because of the crucial role played by the chemical reaction, we must fully resolve the EDL in order to accurately determine $\rho_{C, s}$. The thin-EDL approximation [30] is therefore not possible here. In this Letter we choose parameters that represent silica at $p \mathrm{H}=6.5$, such that $-\log _{10} \rho_{C, b}(M)=6.5, \Gamma=4.6 \mathrm{~nm}^{-2}$, and $p K=6.75$ (an average over the widely varying reported values [38-40]), with millimolar added salt concentrations $\rho_{ \pm, b} \simeq 1 \mathrm{mM}$ such that $\lambda_{D}=10 \mathrm{~nm}$. The single reaction mechanism assumed here is actually too simple to capture the behavior of silica quantitatively, but it serves our purposes here as a generic case. Under these conditions, the equilibrium surface charge and potential are $-e \sigma_{\mathrm{eq}}=-0.069 e / \mathrm{nm}^{2}$ and $\zeta_{\text {eq }}=-93 \mathrm{mV}$. Throughout we set $D=10^{-9} \mathrm{~m}^{2} / \mathrm{s}$ such that $G_{b}=7.5 \mathrm{mS} / \mathrm{m}$ and $G_{s}^{d} \approx 1.2 \mathrm{nS}$ [41]. In agreement with Stern-layer mobilities discussed above, we either set $D_{s}=D$ or $D_{s}=0$ to study presence or absence of Stern-layer conductance, respectively. We furthermore focus on a channel height $H=1 \mu \mathrm{m}$; i.e., $H \gg \lambda_{D}$ and $\mathrm{Du} \simeq 0.16$. Apart from the channel length $L$, the only remaining system parameter is the timescale of the adsorption-desorption process, which will be fitted to experiments below. For computational efficiency we set $k^{\text {des }}=$ $2 \times 10^{-4} \mathrm{~s}^{-1}$ for now, which is comparable to certain photocatalytic rates [42] and comfortably in the reactionlimited regime, as we will see.

In Fig. 1 we show the steady-state field lines of the ionic charge flux $\mathbf{J}_{e}=\sum_{i} z_{i} \mathbf{J}_{\mathbf{i}}$ and a color map of the $x$ component of the electric streaming field $E_{x}$ for a channel of height $H=1 \mu \mathrm{m}$ and total length $2 L=60 \mu \mathrm{m}$, and a pressure drop $\Delta p=0.5$ bar, in Fig. 1(a) without Stern-layer conduction $\left(D_{s}=0\right)$, and in Fig. $1(\mathrm{~b})$ in the presence of both Stern-layer conduction $\left(D_{s}=D\right)$ and charge regulation. The resulting maximum fluid velocity is approximately $0.1 \mathrm{~m} / \mathrm{s}, 3$ orders of magnitude higher than the elecotro-osmotic slip velocity induced by the electric field; i.e., the body forces [last term Navier-Stokes Eq. (2)] are negligible [43]. A striking difference between Figs. 1(a) and 1(b) is nonparallel field lines in Fig. 1(b), even far outside the EDL, and a much weaker electric field especially for $x \in[-L / 2,0]$ in Fig. 1(b). We can trace these two features back to a nonzero surface current $j_{\sigma}(x)$ and a strong heterogeneity of the surface charge profile $\sigma(x)$; both extend over the full width $L$ as shown in Fig. 1(c). This shows that, in addition to the inherent heterogeneities of silica in equilibirum conditions [44], surfaces can exhibit dynamical heterogeneities. We note that the diffusive and conductive contributions to $j_{\sigma}$ [see Eq. (4)] are counteracting and individually 3 orders of magnitude larger than $j_{\sigma}$; i.e., both are essential to obtain this steady state. The near cancellation is the cause of the numerical noise observed for $j_{\sigma}$, and furthermore leads to the surprising conclusion that the effects persist even for $\mathrm{Du} \ll 1$ [45]. Figure 1(b) also shows that $\mathbf{J}_{e}$ and $E_{x}$ depend not only on $z$ but also on $x$, even far outside the EDL. Note that a lateral heterogeneous charge current has also been reported in the case of a (highly conducting) metallic surface [46].

For $\Delta p=0.1 \mathrm{bar}$, the heterogeneous profile $\sigma(x)$ shown in Fig. 1(c) is essentially linear in $x$, locally lower (higher) by about $\pm 25 \%$ of $\sigma_{\text {eq }}$ at the inlet (outlet) side of the chargeable area. For $\Delta p=0.5$ bar; however, $\sigma(x)$ is strongly nonlinear with deviations ranging from $-75 \%$ 
to as high as $+100 \%$ from $\sigma_{\mathrm{eq}}$ at the edges. In equilibrium, such a change in the surface charge would correspond to a $p \mathrm{H}$ varying between 4.9 and 7.4 , i.e., concentrations of $\mathrm{C}^{+}$ that are a factor of 10 higher and lower. The laterally averaged charge in this case decreases to a value as low as $\langle\sigma\rangle=0.7 \sigma_{\text {eq }}$. Additionally, $\langle\zeta\rangle$ also decreases, which causes a breakdown of Eq. (1) [47]. Therefore, the local as well as the average surface charge are not at all (quasi) static quantities, but fully dynamic properties of the solidfluid interface that can be tuned by the fluid flow in the channel. The sharp peaks of $\sigma$ at $x \simeq \pm L / 2$ in Fig. 1(c) are expected in a range of $\lambda_{D}$ next to an uncharged area [48].

We can identify four different timescales that govern the dynamics of this system: (i) the EDL diffusion time $\tau_{\mathrm{dif}}=\lambda_{D}^{2} / D$, which is only about $100 \mathrm{~ns}$ for our parameter choice; (ii) the advection time $\tau_{\mathrm{adv}}=L / u_{x}\left(\lambda_{D}\right)$ for an ion in the EDL to be advected parallel to the surface over a distance $L$, of the order of $\mathrm{ms}$ in all cases studied here; (iii) the conduction time $\tau_{\text {cond }}=L \sigma_{\text {eq }} / j_{\sigma}$ for a charge in the Stern layer to traverse a lateral distance $L$, which is of the order of seconds here; and (iv) the chemical reaction time $\tau_{\text {reac }}=\left(k^{\text {ads }} \rho_{C, S}\right)^{-1}$ [49] of the order of an hour here. We found that significant heterogeneities only occur if $\tau_{\text {reac }}$ exceeds the three others, i.e., if the system is in the reactionlimited rather than in the diffusion-, advection-, or conduction-limited regime. This can be qualitatively understood, e.g., if $\tau_{\text {reac }} \ll \tau_{\text {cond }}$ chemical equilibration would take place before any conductive flux can develop. Note also that $\tau_{\text {cond }} \propto D_{s}^{-1}$ confirms the crucial role played by a finite surface conduction, since $D_{s}=0$ would cause the system to be conduction rather than reaction limited. As long as this ordering of timescales is obeyed and $D_{s} / D=\mathcal{O}(1)$, as noted already on the basis of Refs. $[11,17,18]$, the exact value of $D_{s}$ has no significant effect on the presented results.

So far we have seen that the stationary state of a chargeregulating and conducting surface exposed to a fluid flow becomes heterogeneously charged in a stationary state. In an exciting experiment in 2014, however, the full relaxation dynamics of the surface charge of silica upon an applied water pressure pulse was measured in an experiment that combines microfluidics and sum frequency generation (SFG) [26], albeit only at the central position (here $x=0)$ in the channel. By ruling out alternative interpretations the authors of Ref. [26] attribute their timedependent SFG signal to a time-dependent surface charge $\sigma(x=0, t)$. Here we confirm this interpretation by showing that our theory provides a microscopic explanation for the time dependence of the surface charge, which in the experiments (see inset Fig. 2 or Fig. 2(d) of Ref. [26]) consists of a quasi-instantaneous initial reduction by $40 \%$ (on the timescale of seconds) upon switching on the flow followed by a further reduction by an additional $10 \%$ on the timescale of minutes, and upon switching off the flow a very slow relaxation (on the timescale of tens of minutes)

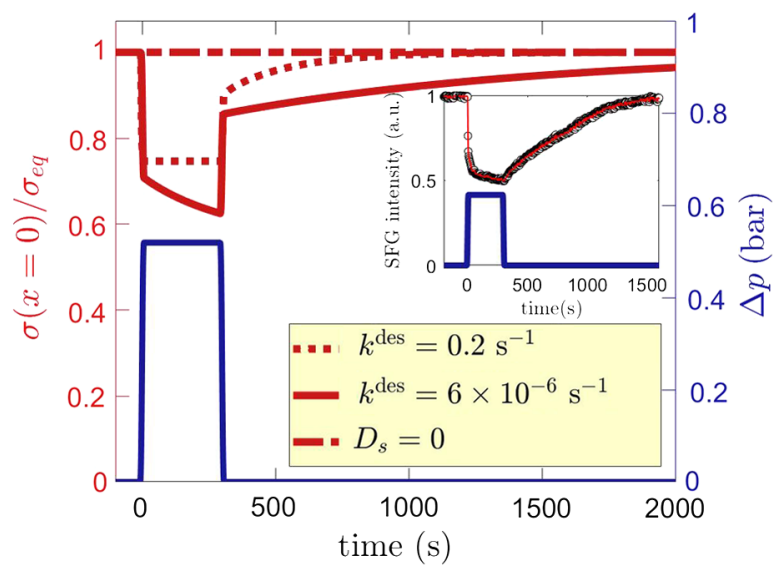

FIG. 2. Time-dependent pressure drop $\Delta p(t)$ (blue) in a channel of dimensions $H=1 \mu \mathrm{m}$ and $L=40 \mu \mathrm{m}$, together with the resulting surface charge $\sigma(x=0, t)$ in the middle of the channel, for a silica surface at $p \mathrm{H}=6.5$ (see text) with desorption rate $k^{\text {des }}=6 \times 10^{-6} \mathrm{~s}^{-1}\left(\tau_{\text {reac }}=1.7 \times 10^{3} \mathrm{~s}\right)$ (red), to be compared with experimental data (in arbitrary units) of Ref. [26] shown in the inset. The red dotted line shows the case of a nonconducting Stern layer with $D_{s}=0$, and the dashed line the case with desorption rate $k^{\text {des }}=0.2 \mathrm{~s}^{-1}\left(\tau_{\text {reac }}=0.05 \mathrm{~s}\right)$.

back to equilibrium. In Fig. 2 we show a time-dependent pressure pulse (blue) similar to the experimental one as well as the surface charge density $\sigma(x=0, t)$ (red) that follows from our theory. Here we use the same silica parameters and bulk concentrations as before in Fig. 1(b), again at $p \mathrm{H}=6.5$ but now with the desorption rate $k^{\text {des }}=$ $6 \times 10^{-6} \mathrm{~s}^{-1}$ as the only "fit" parameter. This corresponds to $\tau_{\text {reac }}=1.7 \times 10^{3} \mathrm{~s}$, which sets the transient behavior of $\sigma(0, t)$. This is also consistent with the observation that $\sigma$ remains constant during such a pressure pulse for larger $\rho_{C, b}$, since $\tau_{\text {reac }} \propto \rho_{C, b}^{-1}$, such that the system is no longer reaction limited for increased counterion concentration. The channel dimensions $H=1 \mu \mathrm{m}$ and $L=40 \mu \mathrm{m}$ are for computational reasons smaller than in the experiment, although the aspect ratio is the same. We checked that this time dependence is hardly dependent on $L$ and $H$ for fixed pressure drop amplitude $\Delta p=0.5$ bar and aspect ratio $L / H=40$ [50]. The similarity between the timedependent experimental SFG signal and $\sigma(x=0, t) / \sigma_{\mathrm{eq}}$ is striking, except perhaps for the strong short-time relaxation immediately after switching off the flow, which is present in our calculations (see Fig. 2) but absent in the experiment (inset). For comparison, Fig. 2 also shows the surface charge for the case of a nonconducting Stern layer with $D_{s}=0$ (dotted red), which is virtually indistinguishable from $\sigma_{\mathrm{eq}}$. By increasing the desorption rate, such that the system becomes less reaction limited, the transient behavior speeds up and the steady state approaches the equilibrium state, as can be observed from the dashed line in Fig. 2 [51].

In conclusion, we apply the classical PNPNS equations (2) to pressure-driven flow through a channel with 
newly formulated boundary conditions for out-ofequilibrium charge regulation and a conducting Stern layer. For realistic system parameters, in particular for silica surfaces, this theory predicts a strong flow-induced heterogeneity of the surface charge and the zeta potential, even for a chemically homogeneous silica surface with $\mathrm{Du} \ll 1$. The traditional Helmholtz-Smoluchowski relation Eq. (1) for the streaming potential, which assumes a laterally constant zeta potential, breaks down for these regulating and conducting surfaces, provided the system is reaction limited; i.e., the chemical reaction is the slowest process. In this reaction-limited regime, a nonzero conductive flux in the Stern layer must be largely compensated by an opposite diffusive surface flux (i.e., by a heterogeneous surface charge) in order to prevent steady-state charge accumulation at the edges due to slow reaction kinetics. The resulting surface charge profile has a reduced lateral average $\langle\sigma\rangle$ compared to equilibrium. Our theory also provides a microscopic picture for measurements on the full time dependence of the relaxation dynamics of the surface charge after switching on and off a tangential flow [26]. We have therefore shown that the combination of a nonzero surface conduction and (s)low chemical adsorption and desorption rates can have dramatic impact on the interpretation of electrokinetics in micro- and nanofluidic experiments, for which the surface charge and zeta potential are a vital component. We expect that these or similar mechanisms also play a role in electro-osmotic and diffusio-osmotic phenomena, which are interesting topics for future research in the context of, e.g., blue-energy harvesting [3,4] and catalysis [52].

We acknowledge fruitful discussions with F. Mugele and I. Siratanu, and thank M. Bonn who kindly provided us with the experimental data of Fig. 2(d) of Ref. [26]. This work is part of the D-ITP consortium, a program of the Netherlands Organisation for Scientific Research (NWO) that is funded by the Dutch Ministry of Education, Culture and Science (OCW). This Letter is a part of an Industrial Partnership Program of the Netherlands Organization for Scientific Research (NWO) through FOM Concept agreement FOM-15-0521. Financial support was provided through the Exploratory Research (ExploRe) programme of BP plc. J. C. E. acknowledges financial support from the European Union's Horizon 2020 programme under the Marie Skłodowska-Curie Grant Agreement No. 795377.

[1] R. T. Wilkin and D. C. DiGiulio, Environ. Sci. Technol. 44, 4821 (2010).

[2] P. W. J. Glover, E. Walker, and M. D. Jackson, Geophysics 77, D17 (2012).

[3] J. Feng, M. Graf, K. Liu, D. Ovchinnikov, D. Dumcenco, M. Heiranian, V. Nandigana, N. R. Aluru, A. Kis, and A. Radenovic, Nature (London) 536, 197 (2016).
[4] A. Siria, P. Poncharal, A.-L. Biance, R. Fulcrand, X. Blase, S. T. Purcell, and L. Bocquet, Nature (London) 494, 455 (2013).

[5] H. Helmholtz, Ann. Phys. (Berlin) 243, 337 (1879).

[6] M. Smoluchowski, Phys. Z. 6, 529 (1905).

[7] J. Lyklema, Fundamentals of Interface and Colloid Science (Academic Press, New York, 1995), Vol. II.

[8] J. Bikerman, Z. Phys. Chem. 163, 378 (1933).

[9] J. J. Bikerman, Kolloid Z. 72, 100 (1935).

[10] J. Overbeek, in Colloid Science I, Irreversible Systems, edited by H. Kruyt (Elsevier, New York, 1952), Chap. 5, pp. 194-244.

[11] J. Lyklema and M. Minor, Colloids Surf. A 140, 33 (1998).

[12] R. Saini, A. Garg, and D. P. J. Barz, Langmuir 30, 10950 (2014).

[13] M. Löbbus, J. Sonnfeld, H. P. van Leeuwen, W. Vogelsberger, and J. Lyklema, J. Colloid Interface Sci. 229, 174 (2000).

[14] J. Sonnefeld, M. Löbbus, and W. Vogelsberger, Colloids Surf. A 195, 215 (2001).

[15] R. O'Brien and W. Rowlands, J. Colloid Interface Sci. 159, 471 (1993).

[16] P. Leroy and A. Revil, J. Colloid Interface Sci. 270, 371 (2004).

[17] M. Minor, A. J. van der Linde, and J. Lyklema, J. Colloid Interface Sci. 203, 177 (1998).

[18] M. Löbbus, H. P. van Leeuwen, and J. Lyklema, Colloids Surf. A 161, 103 (2000).

[19] A. Ajdari, Phys. Rev. Lett. 75, 755 (1995).

[20] A. S. Khair and T. M. Squires, J. Fluid Mech. 615, 323 (2008).

[21] B. W. Ninham and V. Parsegian, J. Theor. Biol. 31, 405 (1971).

[22] D. Y. Chan and D. Mitchell, J. Colloid Interface Sci. 95, 193 (1983).

[23] I. Popa, P. Sinha, M. Finessi, P. Maroni, G. Papastavrou, and M. Borkovec, Phys. Rev. Lett. 104, 228301 (2010).

[24] C. Zukoski and D. Saville, J. Colloid Interface Sci. 114, 32 (1986).

[25] C. S. Mangelsdorf and L. R. White, J. Chem. Soc., Faraday Trans. 86, 2859 (1990).

[26] D. Lis, E. H. G. Backus, J. Hunger, S. H. Parekh, and M. Bonn, Science 344, 1138 (2014).

[27] S. J. Kim, Y.-C. Wang, J. H. Lee, H. Jang, and J. Han, Phys. Rev. Lett. 99, 044501 (2007).

[28] N. Laohakunakorn and U. F. Keyser, Nanotechnology 26, 275202 (2015).

[29] C. B. Picallo, S. Gravelle, L. Joly, E. Charlaix, and L. Bocquet, Phys. Rev. Lett. 111, 244501 (2013).

[30] R. Hunter, Foundations of Colloid Science (Clarendon Press, Oxford, 1992).

[31] See Supplemental Material at http://link.aps.org/ supplemental/10.1103/PhysRevLett.120.264502 for a derivation of the governing equations using density functional theory, including Refs. [32-34].

[32] J. Hansen and I. McDonald, Theory of Simple Liquids (Academic Press, New York, 2013), Vol. IV.

[33] A. Archer, J. Chem. Phys. 130, 014509 (2009).

[34] R. Evans, Adv. Phys. 28, 143 (1979).

[35] R. Kutner, Phys. Lett. A 81, 239 (1981). 
[36] See Supplemental Material at http://link.aps.org/ supplemental/10.1103/PhysRevLett.120.264502 for an analysis of the static limit of the governing equations.

[37] See Supplemental Material at http://link.aps.org/ supplemental/10.1103/PhysRevLett.120.264502 for a more detailed description of the numerical calculations.

[38] T. Hiemstra, J. D. Wit, and W. V. Riemsdijk, J. Colloid Interface Sci. 133, 105 (1989).

[39] C. Macias-Romero, I. Nahalka, H. I. Okur, and S. Roke, Science 357, 784 (2017).

[40] J. Sonnefeld, M. Löbbus, and W. Vogelsberger, Colloids Surf. A 195, 215 (2001).

[41] A. Delgado, F. González-Caballero, R. Hunter, L. Koopal, and J. Lyklema, J. Colloid Interface Sci. 309, 194 (2007).

[42] K. Vinodgopal and P. V. Kamat, Environ. Sci. Technol. 29, 841 (1995).

[43] See Supplemental Material at http://link.aps.org/ supplemental/10.1103/PhysRevLett.120.264502 for an analysis of the governing equations using dimenionsless numbers.

[44] C. Macias-Romero, I. Nahalka, H. I. Okur, and S. Roke, Science 357, 784 (2017).

[45] See Supplemental Material at http://link.aps.org/ supplemental/10.1103/PhysRevLett.120.264502 for a more detailed analysis of the (surprisingly small) role played by the Duhkin number.

[46] J. F. Duval, G. K. Huijs, W. F. Threels, J. Lyklema, and H. P. van Leeuwen, J. Colloid Interface Sci. 260, 95 (2003).

[47] See Supplemental Material at http://link.aps.org/ supplemental/10.1103/PhysRevLett.120.264502 for a more detailed analysis of this important result, which can have significant influence on the interpretation of electrokinetic measurements.

[48] N. Boon and R. van Roij, J. Chem. Phys. 134, 054706 (2011).

[49] See Supplemental Material at http://link.aps.org/ supplemental/10.1103/PhysRevLett.120.264502 for a derivation of this timescale.

[50] See Supplemental Material at http://link.aps.org/ supplemental/10.1103/PhysRevLett.120.264502 for the effect a varying system size (at constant $L / H$ ) has on the steady state and transient behavior.

[51] See Supplemental Material at http://link.aps.org/ supplemental/10.1103/PhysRevLett.120.264502 for more detailed discussion of how the rates influence the resulting steady state behavior.

[52] S. M. Davidson, R. G. H. Lammertink, and A. Mani, Phys. Rev. Fluids 3, 053701 (2018). 